\title{
Tipología de barrios para un aporte metodológico desde la segregación residencial en Zona Metropolitana del Valle de México (ZMVM)
}

\author{
Type of neighborhoods for a methodological contribution from residential segregation in the Metropolitan Zone of the \\ Valley of Mexico (ZMVM)
}

\author{
Ricardo Gómez Maturano ${ }^{1}$ y Ignacio Kunz Bolaños ${ }^{2}$ \\ ${ }^{1}$ Instituto Politécnico Nacional, Ciudad de México, México \\ ${ }^{2}$ Universidad Nacional Autónoma de México, Ciudad de México, México \\ alternz@hotmail.com
}

\section{Resumen}

A pesar de existir un amplio debate sobre la segregación en Latinoamérica existen pocos estudios sobre las ciudades mexicanas, en algunos casos se debe a la resistencia a retomar anglicismos o conceptos que se les señala como descriptivos. Sin embargo, este sesgo ha limitado el conocimiento de procesos urbanos de gran importancia para la comprensión de la ciudad y se ha concluido únicamente por medio de la reflexión que la Zona Metropolitana del Valle de México (ZMVM) es una ciudad segregada. Así la investigación tiene como objetivo explorar una nueva metodología para el estudio de la segregación residencial y desmontar estas conclusiones apriorísticas, utilizando una tipología de barrios que resultan de su homogeneidad: los guetos, enclaves y ciudadelas. Los resultados demuestran que más de la mitad de la población no está aislada y que solamente una parte muy pequeña forma patrones espaciales homogéneos. Si bien, no se puede decir que la segregación es un mito; si se puede asegurar que es mucho menor de lo que se afirma en algunos estudios.

Palabras clave: Segregación residencial, homogeneidad, concentración, gueto, enclave, ciudad de México.

\begin{abstract}
Despite there being a wide-ranging debate about segregation in Latin America, there are few studies on Mexican cities, in some cases it is due to the resistance to use anglicisms or concepts that are referred to as descriptive. However, this bias limited knowledge of urban processes of great importance for the understanding of the city and has been concluded only through reflection that the Metropolitan Area of the Valley of Mexico (ZMVM) is a segregated city. The research aims to explore a new methodology for the study of residential segregation and dismantle these aprioristic conclusions, using a typology of neighborhoods that result from its homogeneity: ghettos, enclaves and citadels. The results show that more than half of the population is not isolated and that only a very small part forms homogeneous spatial pattern. However, segregation cannot be said to be a myth; It is much smaller than what is stated in some studies.
\end{abstract}

Keywords: Residential segregation, homogeneity, concentration, ghetto, enclave, Mexico City.

Documento recibido el 29 de octubre de 2019 y aceptado el 10 de junio de 2020.

Financiado por el Instituto Politécnico Nacional en el proyecto "Expoliación urbana, entre la privación y la esquilmación en la configuración de la segregación residencial de la Zona Metropolitana del Valle de México. Sin fuente de financiamiento”, con número de registro SIP: 20190197.

Cómo citar: Gómez Maturano, R. y Kunz Bolaños, I. (2020). Tipología de barrios para un aporte metodológico desde la segregación residencial en Zona Metropolitana del Valle de México (ZMVM). Revista de Urbanismo, 42, $72-87$. https://doi.org/10.5354/0717-5051.2020.54781 
En los últimos años estudios de las ciudades latinoamericanas apuntan hacia un cambio en "la geografía urbana" (Kaztman et al., 2003), que ha dado paso al surgimiento de una "nueva geografía social" (Prévôt, 2000, p. 405) y en particular se habla de una transformación del patrón de la segregación de la ciudad latinoamericana (Sabatini, Cáceres, y Cerda, 2001). Por ejemplo, en algunas ciudades Chilenas se encontró que la segregación residencial se estaba transformando en dos aspectos: un cambio en su escala geográfica y el aumento de su malignidad (Sabatini, Cáceres, y Cerda, 2001). En una tendencia muy similar, en la ciudad de Montevideo en Uruguay (Kaztman et al., 2003, p. 16), se registró un aumento de la homogeneidad en la composición social de los barrios, así como un correspondiente aumento de la heterogeneidad entre ellos. En Bogotá, Dureau y Salas (2010) encontraron que se redujo la distancia espacial entre los grupos sociales en ciertos sectores de la ciudad; otros trabajos muestran tendencias similares, por ejemplo, en la publicación que coordinan Roberts y Wilson (2009) hay estudios en las ciudades de Buenos Aires, Lima, Montevideo y Sao Paulo que muestran dinámicas similares.

En los estudios de la división socioespacial para la ZMVM se pueden identificar algunas tendencias históricas de la segregación residencial y sus características hasta el año 2005. Para el periodo 1950 - 1970 es clara la consolidación de la distribución de los niveles socioeconómicos, en donde se destacan un sector oriente pobre y un sector poniente de estratos medios y altos. Pero también se identifica una tendencia al aumento en la heterogeneidad interna de las zonas centrales de los niveles económicos altos y una homogeneidad creciente de las zonas de bajos ingresos en el crecimiento periférico (Rubalcaba y Schteingart, 2012).

En el siguiente periodo, 1990 a 2005, continúa la misma tendencia de heterogenización, en la que los residentes de las viviendas con las mejores condiciones redujeron su aislamiento y se vieron expuestos cada vez más a hogares de menores condiciones sociales (Sánchez, 2012, pp. 104-106). Para Aguilar y Mateos (2011, p. 26), lo anterior se debe a las tendencias de dispersión urbana y fragmentación del espacio, generadas por la difusión de los clústeres del "proletariado periférico" y "periferia urbano-rural marginal". Sin embargo, aun con estas tendencias, el grupo socioeconómico más segregado es el de mayor nivel socioeconómico (élites urbanas) y también las clases medias (Aguilar y Mateos, 2011, p. 26), es decir, persiste la alta segregación del estrato con las mejores condiciones habitacionales (Sánchez, 2012, pp. 104-106). Esto va a caracterizar a la ciudad por una concentración de las áreas con mejores condiciones de niveles educativos, vivienda y seguridad social, en el sur, centro y norponiente; y a su alrededor un anillo con una mezcla de estas características; y, por último, en la periferia lejana, principalmente al oriente de la ciudad, la presencia de los niveles más bajos (Pérez-Campuzano y Santos-Cerquera, 2011, pp. 102-103).

Por último, Gómez y Alvarado (2016) identificaron el comportamiento espacial de la concentración y de la exposición de la ZMVM. En donde los grupos socioeconómicos de menores ingresos tienden a estar más concentrados en la medida que viven más cerca del centro de la ciudad; por el contrario, los grupos de altos ingreso tienden a concentrarse mucho más que los de menores ingreso en la medida que su localización es periférica.

Todos estos estudios han ido ofreciendo mapas que describen de la segregación con robustos análisis desde el punto de vista metodológico y de escalas más detalladas; sin embargo, se ha explorado poco otras formas de análisis. Por lo cual, el objetivo de esta investigación es identificar los espacios resultantes de los procesos de agrupamiento, guetos, ciudadelas o enclaves y partir de ahí hacer una análisis sobres las dos principales dimensiones de la segregación residencial, concentración y homogeneidad, para ofrecer nuevas interpretaciones sobre la segregación residencial de la ZMVM.

La investigación parte de la siguiente hipótesis, el estudio de la segregación residencial por medio de la metodología de tipología de barrios a partir de su homogeneidad: los guetos, enclaves y ciudadelas, muestra que la ZMVM no es una ciudad segregada. El primer paso para demostrar esta hipótesis fue construir una clasificación de las tendencias de agrupamiento de los diferentes niveles socioeconómicos y los espacios urbanos que generan, en donde aparecieron los procesos de segregación, congregación y fortificación que han forjado una serie de espacios urbanos como son: el gueto, los enclaves de exclusión o pobreza y las ciudadelas. En segundo lugar, se buscó innovar los mecanismos de 
clasificación de los niveles socioeconómicos, por lo cual se recurrió a los estudios de mercado, que si bien no han tenido un uso extendido en los estudios de segregación y parecen adolecer de un respaldo teórico y empírico para su aplicación en estudios urbanos tienen un gran potencial en busca de una mayor especificidad. Por último, se analizaron dos de las dimensiones más importantes de la segregación residencial, por un lado, la homogeneidad que muestra el grado de aislamiento de los hogares de una misma condición social y por otro lado, la concentración, es decir sobre-representación de un grupo en las unidades espaciales del territorio.

\section{Tipología de barrios y la segregación residencial: guetos,} enclaves y ciudadelas

La segregación residencial se puede conceptualizar como una relación espacial, es decir, la de separación o proximidad territorial entre personas $o$ familias pertenecientes a un mismo grupo social, como sea que este se defina (Sabatini y Sierralta, 2006, p. 4). Por un lado, esta definición que es clara y simple suele complicarse de manera significativa cuando se intenta hacer una lectura de separación territorial de los grupos sociales en la realidad, lo que deriva en una serie de conceptos accesorio y de dimensiones del fenómeno de la segregación lo que, a su vez, lleva a replanteamientos metodológicos cada vez más robustos. Por otro lado, en general los estudios de la segregación en Latinoamérica se han basado en etnografía sobre la vecindad o en identificar marcadores claramente visibles de la segregación, tales como los asentamientos irregulares o las mansiones de los ricos (Roberts, 2010, p. 5), lamentablemente el enfoque cuantitativo sobre la ciudad ha sido marginal en los últimos años en el urbanismo; por lo cual, este análisis busca ser una aportación dentro de los estudios de la segregación residencial.

Dentro de los enfoques cuantitativos se han ofrecido una diversidad de aproximaciones técnicas para el estudio de las diferentes dimensiones de la segregación; sin embargo, hay pocos estudios que identifiquen los procesos de agrupamiento vs los espacios urbanos resultantes. Este será el objetivo principal de este trabajo, en este sentido para Marcuse (2001) el "gueto" es resultado de la segregación, es decir el proceso es la segregación y el espacio urbano resultante es el gueto. Así para este autor el gueto puede ser definido como un área de concentración espacial generada por las fuerzas del grupo social dominante para separar y limitar a un grupo de población particular, definido externamente como racial o étnico o extranjero, considerado como y tratado como inferior por la sociedad dominante (Marcuse, 2001). Esto está en sincronía con lo planteado por Loïc Wacquant (2004, p. 80) donde afirma que el gueto podría ser estudiado de manera más productiva no por analogía con los barrios bajos urbanos, los vecindarios de clase baja y los enclaves de inmigrantes sino junto con la reservación, el campo de refugiados y la prisión, como perteneciente a una clase más amplia de instituciones para el confinamiento forzado de grupos desposeídos y deshonrados. Aunque otros autores hacen notar que es mejor hablar de guetización y no de gueto, con el fin de enfatizar el carácter dinámico del fenómeno, y también para apartarse de definiciones cosistas que conllevan dificultades metodológicas y teóricas (Sabatini, Wormald, Sierralta y Peters, 2008, p. 37) (Figura 1).

\section{Figura 1}

Diagrama de procesos de agrupamiento y sus espacios urbanos

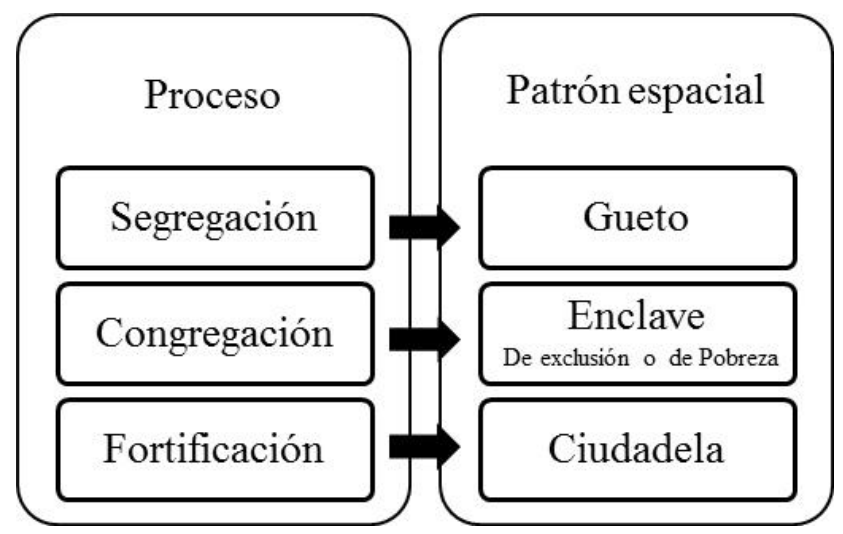

Fuente: elaboración propia con base en los textos de Marcuse (2001), Sabatini, Cáceres, Sierralta y Robles (2008), Sabatini, Wormald, Sierralta y Peters (2008) y Saravi (2004).

El segundo proceso es la congregación y se define como la reunión voluntaria de un grupo de población sin buscar la dominación o la exclusión, es el proceso de formación de un enclave (Marcuse, 2001). Este se puede definir como un área de concentración espacial en la que los miembros de un determinado grupo de población, autodefinido por etnicidad o religión o de otra forma, se 
congregan como un medio para proteger y mejorar su desarrollo económico, social, político y/o cultural (Marcuse, 2001). No obstante, es necesario diferenciar dos tipos de acuerdo a la naturaleza del proceso: el enclave excluyente el cual se define como un área de concentración espacial en la que los miembros de un grupo de población determinado, definido por su posición de superioridad en poder, riqueza o estatus en relación con sus vecinos, se agrupan como medio de proteger esa posición (Marcuse, 2001); el segundo, hace eco de los estudios en Latinoamérica donde está presente la noción de enclaves de pobreza o de pobreza estructural (Saravi, 2004). En este sentido, se identifican dos tipos de enclaves (congregaciones), los de riqueza (excluyente) y los de pobreza (excluido).

El tercer proceso es la fortificación, entendida como la reunión voluntaria de un grupo de población con el propósito de proteger un interés propio dominante y fortalecer esa dominación, es el proceso de formación de una ciudadela. Estas se pueden definir como un área de concentración espacial en la que los miembros de un determinado grupo de población, definido por su posición de superioridad en poder, riqueza o estatus, con relación con sus vecinos, se agrupan como medio de proteger, exhibir y mejorar esa posición (Marcuse, 2001).

Esta tipología de procesos y de patrones espaciales, en combinación con su nivel socioeconómico, resulta en una herramienta conceptual poderosa para el estudio de la división socio espacial; si bien, se reconoce lo que señala Marcuse (2001), en el sentido de que estos procesos y patrones espaciales a menudo comparten las características de más de una de estas definiciones. En esta investigación, se utilizan estas construcciones ideales y se relacionan con las tipologías empíricas de clasificación de unidades o barrios de las ciudades norteamericanas desarrollado por Johnston, Poulsen y Forrest (2003), aunque hay considerar que estas fueron construidas a partir de su perfil étnico (Arriagada, 2008, p. 78) y no con segmentos socioeconómicos como se hace en esta investigación.

El esquema de Johnston, Poulsen y Forrest (2003) reconoce cuatro grandes grupos de acuerdo con el porcentaje que representa el grupo segregado, y al interior de las clases de mayor concentración del grupo segregado, establece dos distintas variantes según lo significativo de la concentración y grado de homogeneidad. En este estudio, se definieron cuatro grandes grupos de acuerdo con el porcentaje del grupo dominante o segregado y en los casos de mayor concentración se dividió en función del nivel socioeconómico (NSE) (Tabla 1):

- Barrio con presencia: son áreas donde el grupo socioeconómico tiene una presencia mayor al porcentaje de la población total de este estrato económico en la ciudad; pero menor al 20 por ciento de los residentes.

- Enclaves no aislados: son barrios donde el grupo socioeconómico representa entre el 20 y 50 por ciento de los residentes, y sus miembros, por tanto, no están aislados de otros grupos, es decir, en el caso de los niveles socioeconómicos altos y medios no buscan la dominación o la exclusión en estas áreas, mientras que, en los bajos, hay interacción con los demás estratos.

- Enclaves: se trata de barrios donde los miembros de los demás niveles socioeconómicos están en minoría y el nivel socioeconómico de estudio representa del 50 al 70 por ciento de los residentes. Estos se pueden dividir en dos, por un lado, los niveles socioeconómicos altos y medios, generan enclaves excluyentes donde se congregan para proteger $y$ mejorar su desarrollo económico, pero muestran un grado de asimilación. Por otro lado, los niveles socioeconómicos bajos son agrupados en enclaves de pobreza; sin embargo, en estos barrios también están en interacción con los demás estratos.

- En el último nivel está la máxima de homogeneidad, son los barrios que reportan 70 por ciento o más del nivel socioeconómico de estudio. Aquí los estratos altos y medios forman ciudadelas con el fin de proteger, exhibir y mejorar esa posición. Por su parte, los niveles socioeconómicos bajos, al ser concentrados en estos barrios, son agrupados en guetos con una alta homogeneidad. 
Tabla 1

Esquema de tipología de barrios por su homogeneidad

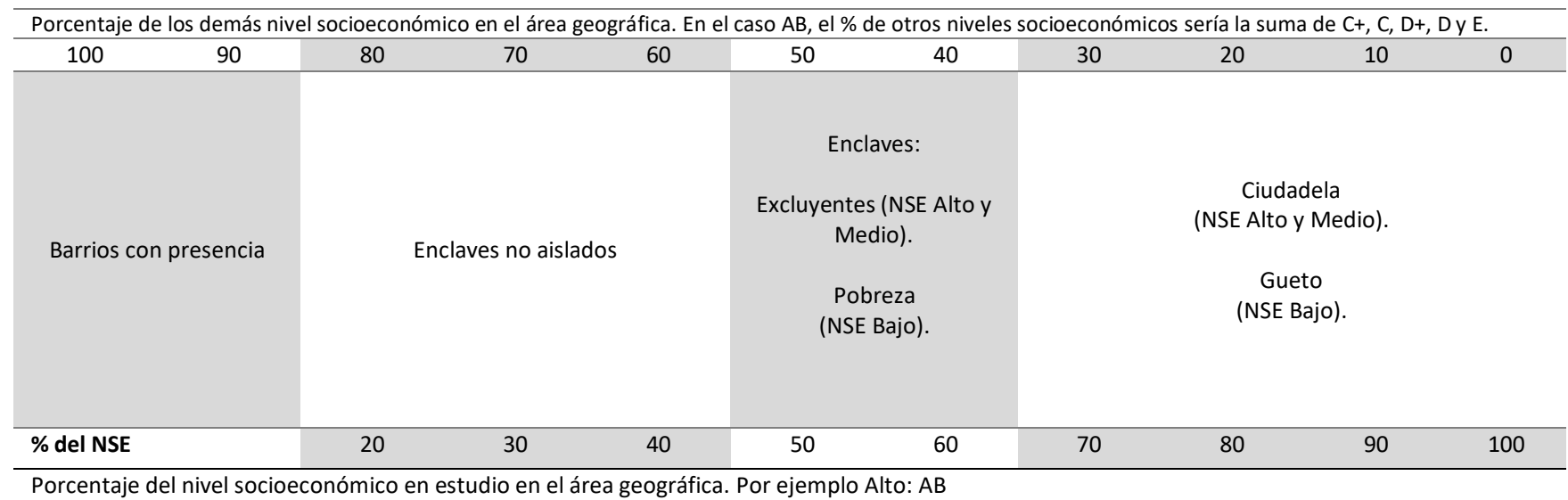

Fuente: elaboración propia con base en la tipología desarrollada por Johnston, Poulsen y Forrest (2003) y de los patrones espaciales descritos por Marcuse (2001), Sabatini, Cáceres, Sierralta y Robles (2008) y Saravi (2004).

\section{Los individuos objeto de estudio en la segregación}

La definición de los procesos y los espacios resultantes del apartado anterior hacen necesario revisan las categorías para llevar a cabo el análisis y la pertinencia de las unidades espaciales, lo que se discute a continuación. Al respecto, entre los diferentes estudios de la segregación y la división socio espacial se recogen cuatro posturas dominantes sobre el grupo de individuos objeto de análisis y la metodología empleada para identificarlos: las que utilizan indicadores institucionales, sobre todo para el estudio de la pobreza; los estudios que operan con una sola variable proxy de la segmentación socioeconómica, como la educación; los que utilizan la variable trabajo, a partir de categorizar las ocupaciones; y por último, los que manejan los estudios de mercado, tanto su clasificación, como su metodología.

La primera postura son los estudios que se basan en indicadores propuestos por dependencias institucionales para la identificación de la pobreza y que los investigadores retoman para el estudio de la segregación. Por ejemplo, Azócar, Henríquez, Valenzuela y Romero en Chile (2008, p. 109) toman como base los indicadores de Necesidades Básicas Insatisfechas (NBI), propuesto por CEPAL, que considera las características de la vivienda, servicios públicos, hacinamiento, personas activas ocupadas y educación del jefe de hogar, carencias físicas de la vivienda y vulnerabilidad.
Otros estudios complementan los índices institucionales, aunque mantienen como eje del estudio las variables de pobreza. Por ejemplo, en Perú, Peters y Skop (2007, p. 157) calculan la segregación utilizando una serie de indicadores que representan varias dimensiones del nivel socioeconómico. Estos incluyen: niveles de educación de la población, tipos de empleo, el estado de arrendamiento de las viviendas ocupadas y el SES (indicador usado en Perú) del hogar, medido por un índice compuesto de pobreza. Una última propuesta es la desarrollada por Pérez y Santos (2011, p. 96) que realizan un análisis factorial inspirado en los estudios utilizados por el CONAPO en México para determinar los grados de marginación urbana.

La segunda postura son los estudios basados a partir de una sola variable proxy de la segmentación socioeconómica. Se basan en una variable de acuerdo con el principio de parsimonia, por ejemplo, en Chile, Garín, Salvo y Bravo (2009, p. 120) tratan de utilizar la mínima cantidad de variables para explicar un fenómeno, explorando los datos de los jefes de hogar con educación superior o la de hogares con tenencia de vehículo. Otros estudios con una variable son los que realizan una descomposición de ésta. Por ejemplo, en Argentina los investigadores Groisman y Suárez por el nivel educativo del jefe de hogar, (2006, p 28). Esta decisión se debe en algunos casos a que no se dispone de datos censales sobre 
los ingresos, así también en Argentina, Molinatti (2013, p. 117) utiliza como variable proxy de la segmentación socioeconómica el máximo nivel educativo alcanzado por el jefe de hogar.

Otros estudios de una sola variable la descomponen en dos grupos con el fin de estudiar grupos socioeconómicos extremos. Por ejemplo, en Brasil y Chile, los autores Thais, Bichir y Moller-Holtkamp (2008, p. 49) observan la distribución de los grupos de población, clasificándolos en población responsable sin instrucción y población responsable con alta escolaridad. El retomar grupos extremos es apropiado, de acuerdo con los investigadores argentinos

La tercera postura utiliza la variable de ocupación de las personas, por ejemplo, en Argentina, Linares (2013, p. 29-30) utiliza como atributo de clasificación y estratificación social la 'calificación de las ocupaciones' propuesta por el Instituto Nacional de Estadísticas y Censos en base al Clasificador Nacional de Ocupaciones. Este investigador busca un atributo de las ocupaciones y no de las personas, que califica la complejidad de las tareas que indefectiblemente se encontrarán asociadas a una remuneración correspondiente. Otros investigadores como Link, Valenzuela y Fuentes (2015, p. 158) en Chile, señalan que la variable ocupacional resulta pertinente para un estudio sobre la estructura y composición socioterritorial.

La última postura, son los estudios basados a partir de la clasificación y la metodología de los estudios de mercado. En esta visión, los estratos sociales se distinguen siguiendo las ideas del marketing. Así, por ejemplo, en Chile Sabatini, Wormald, Sierralta y Peters (2008, p. 23) trabajaron con cinco grupos de hogares, que representan aproximadamente las siguientes proporciones de la población de la ciudad: ABC1 o elites (10\%), C2 estrato medio alto (20\%), C3 o estrato medio (25\%), D o estrato bajo (35\%) y $\mathrm{E}$ o estrato muy bajo (10\%). Los estratos los construyeron combinando dos puntajes: educación del jefe de hogar y posesión de un conjunto de diez bienes.

Cada una de las cuatro posturas para definir los grupos de individuos objeto de análisis y sus metodologías empleadas tienen sus ventajas y desventajas. Entre éstas se identifican las siguientes: en primer lugar, los estudios que utilizan indicadores institucionales suelen usar como base índices de pobreza o marginación, lo que tiene varias desventajas entre las que destacan dos: primero, por el tipo de variables utilizadas es posible que discriminen bien la parte baja del espectro socioeconómico, pero suelen ser muy ineficaces para identificar los individuos u objetos en las zonas de medianos y altos ingresos; y segundo, cuando se utiliza información censal, como en el caso de México, los empleados domésticos que suele vivir en las viviendas de los grupos de mayor ingreso, son censada y contribuyen a sesgar el valor de la zona, e incluso, de la familia/vivienda.

En segundo lugar, los estudios basados en una sola variable asumen un riesgo importante al suponer que esa variable es, en todos los casos, un buen reflejo del estatus socioeconómico. En México, por ejemplo, las variables asociadas a educación son un buen indicador para estudios regionales que incluyen áreas urbanas y rurales; sin embargo, su aplicación a la ciudad es mucho más limitada, ya que las facilidades de educación pública superior han permitido que una proporción significativa de población joven de escasos recursos tenga acceso a ella.

En tercer lugar, los que utilizan la variable trabajo a partir de categorizar ocupaciones, tienen el problema de que los ingresos y el estatus socioeconómico puede variar mucho para las mismas ocupaciones, además de la dificultad para clasificar a algunos trabajadores que se encuentran fuera del mercado laboral formal que, en el caso de Latinoamérica, es significativamente alto.

Por último, los estudios de la segregación y los estudios de mercado no han tenido un uso extendido en otras geografías y parecen adolecer de un respaldo teórico e empírico para su aplicación en estudios urbanos. Sin embargo, tienen un gran potencial en el contexto de los estudios de la división socio espacial de los profesionales del espacio geográfico en México en busca de una mayor especificidad en los estudios de la segregación.

\section{La segregación residencial en al ZMVM y sus aspectos metodológicos}

Los estudios sobre segregación utilizan como objeto de estudio los grupos resultado de indicadores de pobreza generados por instituciones gubernamentales. En estos estudios existe un claro predominio de análisis factorial como técnica para delimitar los niveles de estratificación socioeconómica (Ariza y Solís, 2009, p. 174); sin embargo, 
estos estudios tienen una gran desventaja, ya que homogenizan o mediatizan las unidades territoriales de las que se parte, tradicionalmente las áreas geoestadísticas básicas (AGEB's) las cuales pueden llegar a mostrar contrastes significativos en su composición socioeconómica. Por esta razón es fundamental usar metodologías que permitan estudiar la heterogeneidad / homogeneidad interior de las unidades territoriales que se usan de base.

La principal razón por la cual en México se utilizan las técnicas de análisis factorial para clasificar los estratos socioeconómicos, es la disponibilidad de numerosas variables censales en el Instituto Nacional de Estadística y Geografía (INEGI) que teóricamente se asocian al nivel socioeconómico. Pocos estudios han tenido acceso a datos más específicos. Ariza y Solís (2009) trabajaron con bases de datos especialmente preparadas por el INEGI, en las que se incluye agregados de información no contenidos en los tabulados convencionales. Otra de las razones que explican la utilización del análisis factorial es el limitado acceso a los micro datos, solamente existe el antecedente, en el tema de segregación, el estudio de Rodríguez (2008, p. 56) que, a partir del acceso a micro datos construyó categorías con dos variables.

En este sentido, el presente estudio se benefició del acceso al laboratorio de microdatos del INEGI, lo que permitió usar las clasificaciones y metodologías de los estudios de mercado para la identificación de los niveles socioeconómicos de los jefes de familia y reconocer su distribución precisa en la ZMVM, que lamentablemente, no estaba disponible hasta hace unos pocos años. La ventaja de contar con los micro datos es que permite una explotación mucho más flexible de las variables, admite todo tipo de análisis estadísticos y lo más importante, permite relacionar variables que no siempre se pueden cruzar con los datos agregados de los censos de población ${ }^{1}$.

El primer paso para determinar el nivel socioeconómico (NSE) del jefe de familia fue identificar, en los microdatos del Censo de Población y Vivienda del 2010, las frecuencias de cada una de las variables definidas en el universo. Tales variables fueron: agua entubada dentro de la vivienda, piso de madera, mosaico $\mathrm{u}$ otro recubrimiento, existencia en la vivienda de refrigerador, lavadora, auto propio, televisión, teléfono, computadora o internet. Utilizando estos datos se elaboró una tabla donde se indicaba las proporciones de tenencia y de escasez (tasa de penetración) para el año 2010, posteriormente se obtuvo un ponderador con relación al grado de escasez de cada variable con base 1000. Lo anterior quiere decir que aquellos hogares que tengan presencia para todas las variables (tienen todos los bienes o servicios), obtendrán el valor máximo que es 1000 , mientras que aquello hogares que no tengan ningún de estos bienes o servicios obtendrán valor 0 (Tabla 2 ).

$$
\mathrm{J}=\frac{\Sigma \mathrm{BI} \times \mathrm{Y}}{\Sigma \mathrm{BN}}
$$

Donde:

- $\Sigma$ Bi es la escasez de cada variable,

- $\quad \Sigma B n$ es la sumatoria de las tasas de escasez de todas las variables,

- $\quad$ Y es el ponderador de grado de escasez con base en 1000,

- J es el ponderador para las diferentes variables sobre bienes y servicios.

\footnotetext{
${ }^{1}$ Esta metodología fue construida durante una pasantía para profesor extranjero en el Centro de Desarrollo Urbano Sustentable de la Pontificia Universidad Católica de Chile, en el proyecto de investigación
}

"Gentrificación de barrios populares: amenaza de desplazamiento, potencial de integración e implicancias de política urbana en Santiago de Chile y Ciudad de México", coordinado por el Dr. Francisco Sabatini. 
El segundo paso para determinar el nivel socioeconómico del jefe de familia, fue identificar las frecuencias de los niveles educativos alcanzado por los jefes de hogar, para determinar la escasez acumulada de los distintos niveles de instrucción del jefe de hogar. Los jefes de hogar sin educación obtienen un valor 0 , mientras que los jefes de hogar con niveles universitarios completos o más obtienen valor 1000. La fórmula utilizada es la siguiente:

$$
\mathrm{X}=\frac{\sum \mathrm{aNi} \times \mathrm{Y}}{\Sigma \mathrm{Ns}}
$$

Donde:

- $\Sigma a N i$ es la escasez acumulada para un nivel de instrucción dado,

- $\quad$ NNs es la escasez del nivel superior,

- $\mathrm{Y}=$ ponderador con niveles universitarios completos $\mathrm{y}$ más, con base en 1000,
- $X=$ es el ponderador para los distintos niveles de instrucción del jefe de hogar.

El tercer paso, con los puntajes obtenidos por los jefes de hogar se procedió a asignar en la base de los microdatos los valores de cada uno de los ponderados a cada una de las variables; segundo, se realizó una sumatoria por cada jefe de familia de cada uno de los ponderados; y por último el resultado de esta sumatoria se dividió entre 2.

El cuarto paso, fue la clasificación de los distintos niveles socioeconómicos, estableciendo para tal fin los percentiles propuestos por la Asociación Mexicana de Agencias de Investigación de Mercados y Opinión Pública (AMAI), estimadas a partir de los estudios realizados por dos empresas asociadas a AMAI: IBOPE/AGB y Nielsen (López, 2009). Ambas empresas utilizan una muestra nacional representativa como marco muestral de sus estudios panel de audiencia y consumo respectivamente.

\section{Tabla 2}

Niveles socioeconómicos de la Zona Metropolitana de la Ciudad de México por valor ponderado para el año 2010.

\begin{tabular}{lcccc}
\hline Estrato & Nivel socioeconómico & \% AMAI 2008 para la ZMVM & Suma de porcentaje & Valor del ponderado \\
\hline Alto & A/B & 6,1 & 100 & 999,01 y más \\
Medio-alto & C+ & 12,2 & 93,9 & $830,51-999,00$ \\
Medio & C & 16,4 & 81,7 & $612,51-830,50$ \\
Bajo superior & D+ & 38,2 & 65,3 & $252,01-612,50$ \\
Bajo & D & 18,8 & 27,1 & $87,01-252,00$ \\
Muy bajo & E & 8,3 & 8,3 & $0,00-87,00$ \\
\hline
\end{tabular}

Fuente: elaboración propia con datos construidos en el laboratorio de microdatos del INEGI con datos del Censo de Población y Vivienda del 2010. Así se determinó los niveles socioeconómicos al cual pertenecen cada uno de los jefes de familia en función al puntaje obtenido y a los percentiles para la ZMVM desarrollados por el AMAI (sobre los percentiles ver López, 2009). 
En este sentido, un jefe de familia clasificado como Alto debió haber alcanzado un mínimo de 999 puntos de 1000, así que tenía estudios de licenciatura o mayores, tenía agua entubada dentro de la vivienda, piso de madera, mosaico u otro recubrimiento, refrigerador, lavadora, auto propio, televisión, teléfono, computadora e internet. Por el contrario, un jefe de familia clasificado como Muy Bajo debió haber alcanzado un máximo de 87 puntos de 1000 , así que solo contaba con estudios de primaria o preescolar, un televisor, un refrigerador y agua entubada.

\section{Los espacios de la homogeneidad, la búsqueda de ser mayoría a pesar de ser minoría.}

La primera dimensión por estudiar es la homogeneidad, que mide la probabilidad de que un miembro de un grupo comparta la misma unidad espacial con otro individuo de su mismo grupo. Este análisis muestra el grado de aislamiento de los hogares de una misma condición social; sin embargo, no se utilizaron los índices convencionales, sino que se localizaron y clasificaron los espacios que conforman las dinámicas de la división socio espacial para estudiar la homogeneidad: barrios con presencia, enclaves no aislados, enclaves (pobreza y riqueza), y los guetos o ciudadelas ( $\underline{\text { Tabla } 3}$ ).

En conjunto el nivel socioeconómico que genera espacios más homogéneos son el estrato más Alto $(A B)$, aunque todos ellos representan poco en la ciudad. El primero es resultado de los procesos de fortificación y de congregación por parte de las niveles socioeconómicos más ricos, es decir, es consecuencia de una reunión voluntaria con el propósito de proteger sus intereses y fortalecer su dominación, llegando a crear dos tipos de espacios: las ciudadelas, donde el NSE representa más del $70 \%$ de todos los jefes de familia del AGEB, pero solo viven el 1,6\% de los jefes de familia de este estrato, alrededor de 7.052 jefes de familia; y los enclaves de exclusión, donde son mayoría al representar entre el 50 y $70 \%$ de los jefes de familia, aquí vive $11,5 \%$ de los jefes de familia clasificados en este nivel, no llegando a ser 50.000 familias (Figuras 2 y $\underline{3}$ ).

\section{Tabla 3}

Distribución de la población en hogares por niveles socioeconómicos según AGEB's clasificados por la tipología de Barrios adaptada de Johnston et al.

\begin{tabular}{|c|c|c|c|c|c|c|c|c|c|c|c|c|}
\hline \multirow{2}{*}{$\begin{array}{l}\text { Espacio/ } \\
\text { nivel socio- } \\
\text { económico }\end{array}$} & \multicolumn{2}{|c|}{$A B$} & \multicolumn{2}{|c|}{ C+ } & \multicolumn{2}{|c|}{ C } & \multicolumn{2}{|c|}{$D+$} & \multicolumn{2}{|c|}{ D } & \multicolumn{2}{|c|}{$\mathbf{E}$} \\
\hline & $\%$ & $\#$ & $\%$ & $\#$ & $\%$ & $\#$ & $\%$ & $\#$ & $\%$ & \# & $\%$ & \# \\
\hline $\begin{array}{l}\text { Ciudadelas / } \\
\text { Guetos }\end{array}$ & 1,6 & 7.052 & 0,0 & 0 & 0,0 & 0 & 0,1 & 2.070 & 0,0 & 94 & 0,0 & 376 \\
\hline $\begin{array}{l}\text { Enclaves de } \\
\text { exclusión / } \\
\text { pobreza }\end{array}$ & 11,5 & 48.339 & 0,0 & 31 & 0,0 & 119 & 8,5 & 167.934 & 0,1 & 1.086 & 1,0 & 4.086 \\
\hline $\begin{array}{l}\text { Enclaves no } \\
\text { aislados }\end{array}$ & 45,7 & 190.999 & 39,6 & 201.829 & 51,7 & 441.727 & 88,2 & 1.739 .068 & 69,2 & 604.131 & 23,2 & 95.015 \\
\hline $\begin{array}{l}\text { Barrios con } \\
\text { presencia }\end{array}$ & 25,5 & 106.746 & 25,7 & 130.963 & 16,7 & 142.773 & 0 & 0 & 4,3 & 38.223 & 52,7 & 215.195 \\
\hline Menor a su \% & 15,4 & 353.136 & 34,6 & 332.823 & 31,4 & 584.619 & 3,1 & 1.909 .072 & 26,2 & 643.534 & 22,9 & 314.672 \\
\hline
\end{tabular}

Fuente: elaboración propia datos construidos en el laboratorio de microdatos del INEGI utilizando el Censo de Población y Vivienda del 2010, con la tipología de barrios adaptada de Johnston, Poulsen, y Forrest (2003) y de los patrones espaciales descritos por Marcuse (2001), Sabatini, Cáceres, Sierralta y Robles (2008) y Saravi (2004). 


\section{Figura 2}

Ciudadelas, enclaves y barrios con presencia de estratos Alto (AB) y Medio-Alto (C+)
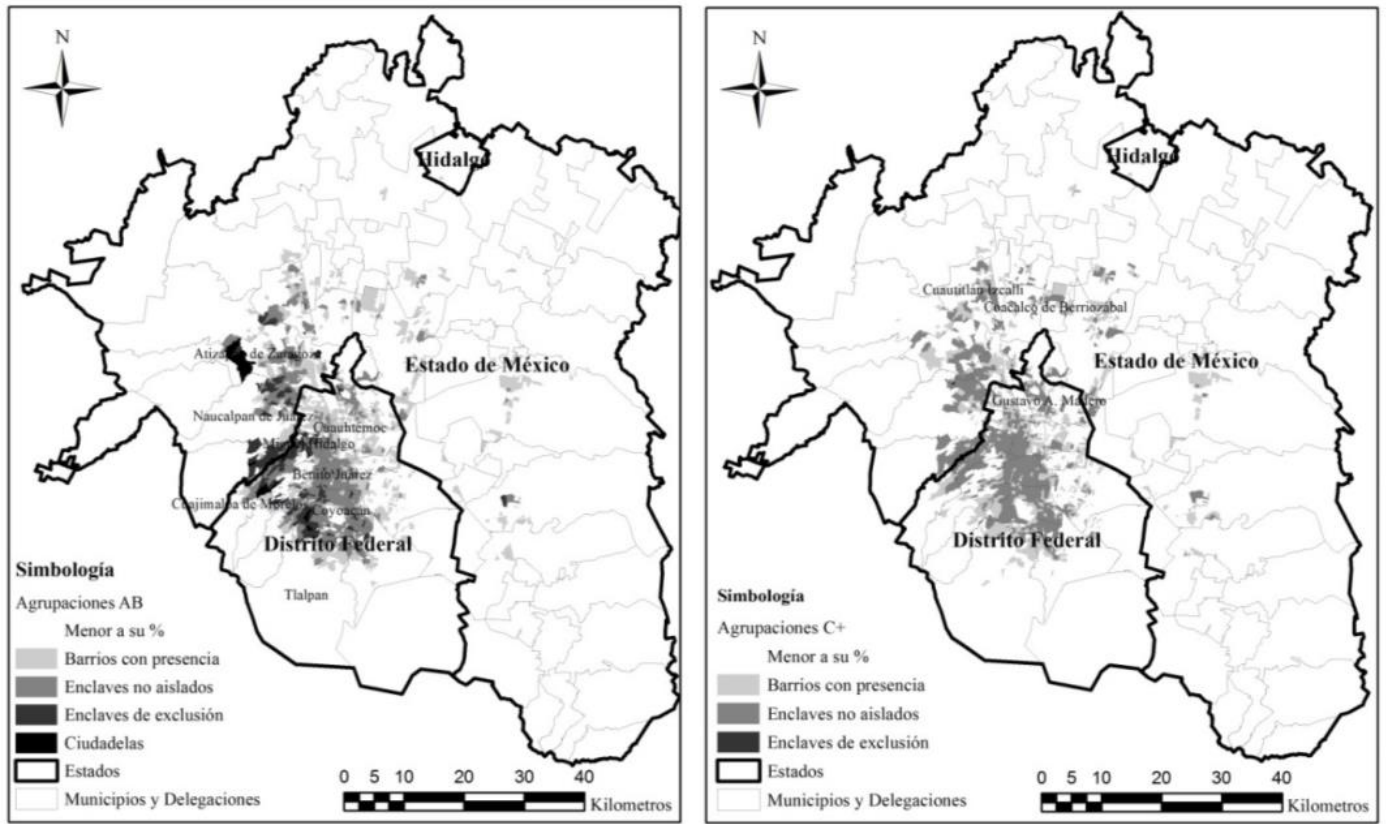

Fuente: elaboración propia, datos por AGEB construidos en el laboratorio de microdatos del INEGI utilizando el Censo de Población y Vivienda del 2010.

\section{Figura 3}

Guetos, enclaves y barrios con presencia de estratos Medio (C) y Bajo-superior (D+)
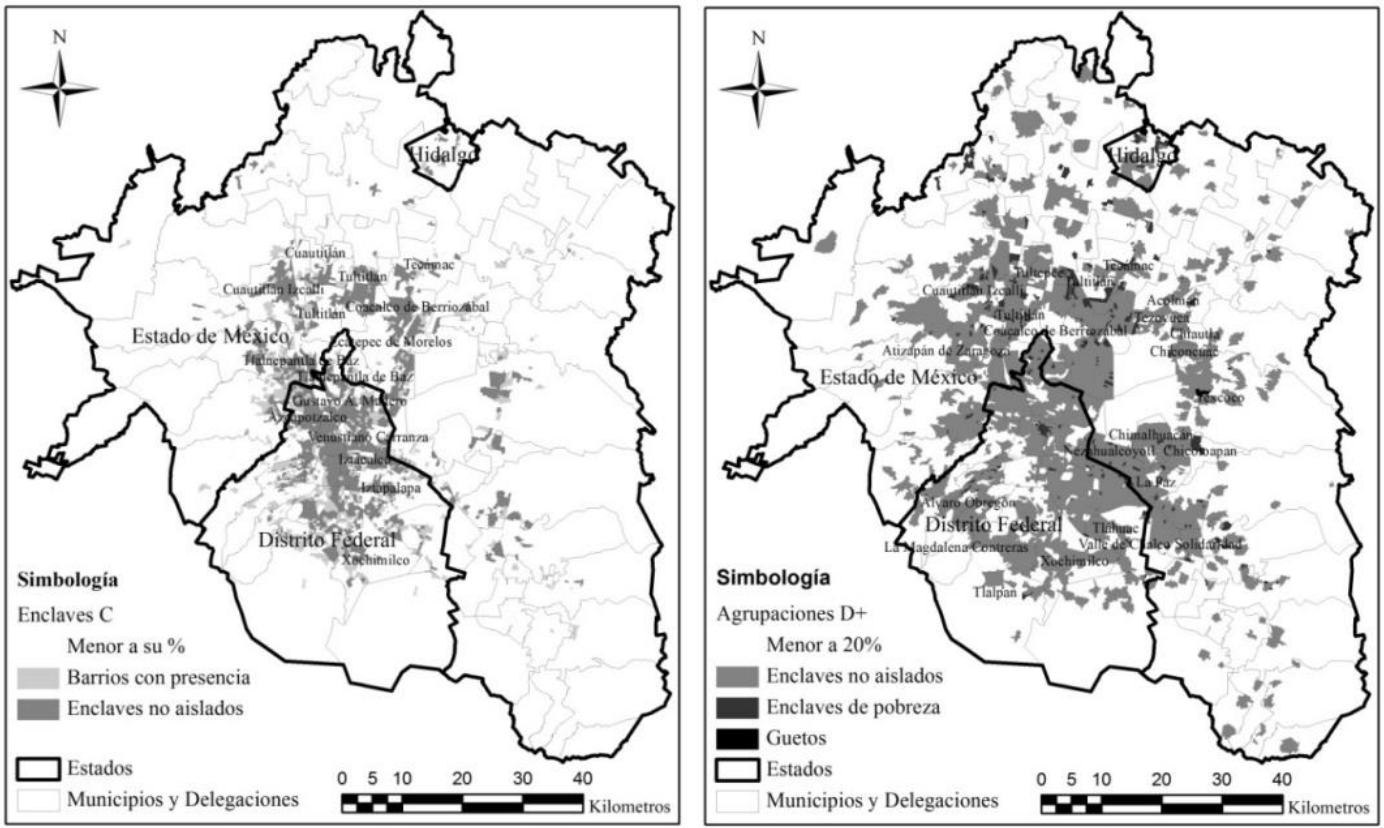

Fuente: elaboración propia, datos por AGEB construidos en el laboratorio de microdatos del INEGI utilizando el Censo de Población y Vivienda del 2010. 
El segundo NSE que genera espacios de homogeneidad es el estrato Bajo Superior se explica por una cuestión de volumen de población, ya que este estrato representa casi el $40 \%$ de las familias de la ZMVM, así que la congregación no es el resultado de una búsqueda por parte de este grupo, de hecho, solo están en enclaves de pobreza el $8,5 \%$ de los jefes de familia del estrato, cerca de 167.934 jefes de familia. Por último, los guetos y enclaves de pobreza del estrato Muy Bajo son resultado del proceso de segregación espacial generada por las fuerzas de demanda de vivienda del grupo social dominante, que los separa y limita la oferta de vivienda, generando procesos de agrupación que no han llegado a ser significativos, solo vive en estos espacios poco más del $1 \%$ de las familias de este estrato, es decir solo 4.000 familias. Así los datos muestran que es poco el porcentaje de las familias de la ZMVM que habita en condiciones de gueto, en particular el Bajo Superior y el Muy Bajo (Figuras 2 y $\underline{3}$ ).

Los enclaves no aislados, o también llamados enclaves de inclusión, son los barrios donde viven más hogares en la ZMVM, de hecho, son los tipos de ocupación más frecuentes en todos los estratos con excepción del Bajo. En estos enclaves sus miembros no están aislados de otros grupos, así los niveles socioeconómicos altos no buscan la exclusión y los bajos se encuentran en interacción con los demás estratos. Por una parte, los estratos de mayores ingresos viven en enclaves no aislados, por ejemplo el Alto con el $45,7 \%$ de sus familias, es decir aproximadamente 190.000, Medio Alto con 201.829 familias, representando el 39,6\% y por último, el Medio con 441.727 familias, que representan el $51,7 \%$ del estrato; por otra parte, los estratos de menores ingresos se pueden dividir en dos grupos, por un lado, el Bajo Superior y el Bajo, muestran una tendencia mayor de vivir en estos barrios, el primero con más de 1.700 .000 representando el $88,2 \%$ y el segundo con 604.131 familias, que representa el 69,2\% de los hogares de estos estratos en estos espacios de heterogeneidad. Por otro lado, en el estrato Muy Bajo, al contrario de todos los demás estratos económicos, los jefes de familia son los que tienen un menor porcentaje de hogares que habitan en estos barrios, solo el $23,28 \%$ del total viven en enclaves no aislados, poco más de 95.000 familias.

Por último, en cuanto a los barrios con presencia, en donde son minoría los diferentes niveles socioeconómicos se pueden dividir en tres grupos. Los jefes de familia de mayores ingresos, Alto, Medio Alto y Medio, vive el 25,5\%, $25,7 \%$ y $16,7 \%$ respectivamente de sus hogares en estos espacios. Los jefes de familia del nivel socioeconómico Bajo Superior y Bajo son poco significativos en estos barrios debido a que su porcentaje de población en el AGEB no alcanzó el $20 \%$ que es el límite de clasificación para los barrios con presencia, por lo cual fueron clasificados en enclaves no aislados o barrios con una presencia mínima (menor a su porcentaje). Por último, en el estrato Muy Bajo casi la mitad de sus jefes de familia viven distribuidos como minorías en estos barrios con presencia, dicho de otra forma, la mitad de los más pobres son minorías que se mezclan con otros estratos socioeconómicos.

En síntesis, los niveles socioeconómicos de mayores ingresos generan procesos de concentración y fortificación, procurando vivir en enclaves de exclusión o ciudadelas donde son mayoría y casi no viven en barrios donde son minoría; y por el contrario, los estratos Muy Bajos tienden a vivir más en barrios donde son minoría.

\section{La ciudad de México entre la alta concentración y el desvanecimiento de sus grupos socioeconómicos}

Otra de las dimensiones de la segregación es la concentración de los grupos sociales, esta es la sobrerepresentación o subrepresentación de un grupo en las unidades espaciales (Apparicio, 2000). Para ello, se identifica la proporción del territorio (AGEB's) en los que están presentes los jefes de familia de los diferentes niveles socioeconómicos según el patrón espacial de ocupación (Ver tabla No. 4). El nivel socioeconómico de mayores ingresos $A B$ muestra las siguientes dinámicas, primero es el que genera más espacios homogéneos, con sus ciudadelas y enclaves de exclusión, de todos los niveles económicos y muy concentrados en pocos barrios: por un lado, en las ciudadelas, vive el 1,69\% de los jefes de familia de este nivel ocupando solo el $0,34 \%$ de las AGEB's. Por otro lado, 1 de cada diez jefes de familia de este nivel viven en enclaves de exclusión donde están en mayoría, sin embargo, solo ocupan el 2,37\%. Segundo, casi 1 de cada 2 jefes de familia de este nivel socioeconómico se congrega en enclaves no aislados, es decir no buscan la dominación o la exclusión al habitar en espacios en los que solo representan entre el 20 y el $50 \%$ de los hogares, aunque están concentrado en el 11,64\%. Finalmente, los 
jefes de familia de estos niveles socioeconómicos viven en AGEB's en condiciones de poca o mínima presencia. Se trata de un grupo social que muestra dos comportamientos muy disímiles fuerte concentración por un lado y, una alta dispersión por el otro. ¿Cómo explicar esta última? Puede ser la mejora social de algunas familias en barrios de clase media o baja, o bien, las familias residuales de antiguos barrios de nivel alto que permanecen después de un proceso de sucesión (ver Tabla 4 y Figura 2).

El nivel socioeconómico Medio Alto no ha generado espacios de alta homogeneidad, la mayor parte de los barrios donde viven son enclaves no aislados y barrios con presencia; pero se encuentran concentrados solo en algunos espacios de la ZMVM. Por ejemplo, 4 de cada diez jefes de familia de este nivel vive en enclaves no aislados, pero concentrados en el 16,63\% de AGEB's de la ZMVM. Este grupo, con poca homogeneidad y fuerte concentración, puede explicarse por los procesos de sucesión, se trata de barrios antiguos de clase media alta que han ido desvalorizándose permitiendo la llegada de hogares de menor estatus. Por otra parte, un $25,7 \%$ de estos jefes de familia del Medio Alto viven en barrios con presencia (12,2 a $20 \%$ de los jefes de familia) ocupando solamente el $14 \%$. Similar al estrato económico anterior cerca del 70\% de los AGEB's tienen mínima presencia de este nivel socioeconómico, en ellos se distribuye el 34,6\% de las familias de estrato Medio Alto, que se puede afirmar, viven dispersas y mezcladas (ver Tabla 4 y Figura 2).

El estrato económico Medio tampoco ha generado espacios de alta homogeneidad, la mayor parte de los barrios donde viven son heterogéneos, en enclaves no aislados, en barrios con presencia y se encuentran desconcentrados a lo largo de la ciudad. Primero, la mitad de los jefes de familia de este estrato viven en enclaves no aislados y se encuentra dispersos en el 30\% de los AGEB's. Segundo, el otro casi $50 \%$ de jefes de familia vivía en barrios con presencia significativa y mínima (porcentajes menores al $16,4 \%$ ) y se distribuían en el $68 \%$ de las áreas estadísticas. Aunque es importante señalar que, a diferencia de los estratos de mayores ingresos ( $A B$ y $C+$ ) éste está ligeramente más concentrado. En otras palabras, lo grupos más ricos pueden estar dispersos por la ciudad con una presencia mínima, conviviendo con otros estratos sociales, igual, como se verá más adelante, el grupo más pobre, lo que significa que una parte de los estratos extremos tienden a convivir con otros grupos (ver Tabla 4 y Figura 3).

\section{Tabla 4}

Distribución de la población y AGEB clasificados por la tipología de Barrios.

\begin{tabular}{|c|c|c|c|c|c|c|c|c|c|c|c|c|}
\hline \multirow{2}{*}{$\begin{array}{l}\text { Espacio/ } \\
\text { nivel socio- } \\
\text { económico }\end{array}$} & \multicolumn{2}{|r|}{$A B$} & \multicolumn{2}{|c|}{$\mathrm{C}+$} & \multicolumn{2}{|c|}{ C } & \multicolumn{2}{|c|}{ D+ } & \multicolumn{2}{|r|}{ D } & \multirow{2}{*}{\multicolumn{2}{|c|}{$\begin{array}{l}\text { E } \\
\text { \% AGEB }\end{array}$}} \\
\hline & $\% \mathrm{JF}$ & $\%$ AGEB & $\% \mathrm{JF}$ & $\%$ AGEB & $\% \mathrm{JF}$ & $\%$ AGEB & $\% \mathrm{JF}$ & $\%$ AGEB & $\% \mathrm{JF}$ & $\%$ AGEB & & \\
\hline $\begin{array}{l}\text { Ciudadelas / } \\
\text { Guetos }\end{array}$ & 1,69 & 0,34 & 0,00 & 0,00 & 0,00 & 0,00 & 0,11 & 0,65 & 0,01 & 0,18 & 0,09 & 0,12 \\
\hline $\begin{array}{l}\text { Enclaves de } \\
\text { exclusión / } \\
\text { pobreza }\end{array}$ & 11,57 & 2,37 & 0,01 & 0,05 & 0,01 & 0,05 & 8,52 & 7,53 & 0,12 & 0,90 & 1,00 & 1,40 \\
\hline $\begin{array}{l}\text { Enclaves no } \\
\text { aislados }\end{array}$ & 45,72 & 11,64 & 39,64 & 16,63 & 51,78 & 32,03 & 88,27 & 75,79 & 69,20 & 43,97 & 23,28 & 11,34 \\
\hline $\begin{array}{l}\text { Barrios con } \\
\text { presencia }\end{array}$ & 25,55 & 17,28 & 25,72 & 14,84 & 16,74 & 14,22 & 0,00 & 0,00 & 4,38 & 3,33 & 52,72 & 28,99 \\
\hline Menor a su \% & 15,47 & 68,38 & 34,64 & 68,48 & 31,46 & 53,70 & 3,10 & 16,02 & 26,28 & 51,63 & 22,91 & 58,15 \\
\hline
\end{tabular}

Fuente: elaboración propia datos construidos en el laboratorio de microdatos del INEGI utilizando el Censo de Población y Vivienda del 2010, con la tipología de barrios adaptada de Johnston, Poulsen y Forrest (2003) y de los patrones espaciales descritos por Marcuse (2001), Sabatini, Cáceres, Sierralta y Robles (2008) y Saravi (2004). 
El nivel socioeconómico Bajo Superior al ser el grupo socioeconómico más numeroso si ha generado enclaves de pobreza de alta homogeneidad; sin embargo, la mayor parte de estos viven en enclaves no aislados y desagrupado en toda la metrópoli. Primero, menos de 1 de cada 10 diez jefes de familia de este nivel viven en enclaves de pobreza en el 7,5\% de los AGEB's. Esto es, aunque los hay, son pocas las familias del estrato que viven en homogeneidad y concentrados. Segundo, casi el $90 \%$ de estos jefes de familia viven en enclaves no aislados y desconcentrados en el $76 \%$ de los AGEB's. Por último, casi no habitan barrios en los que no sean mayoría, en el $16 \%$ de los barrios de la ciudad tienen poca o nula presencia. Se podría afirmar la que familias de estratos Bajo Superior representan a la ZMVM (ver Tabla 4 y Figura 3).

El estrato Bajo no ha generado espacios de alta homogeneidad, la mayor parte de los barrios donde viven son heterogéneos, en enclaves no aislados, en barrios con presencia y se encuentran desconcentrados en la ZMVM. Por ejemplo, 7 de cada 10 jefes de familia del estrato Bajo viven en enclaves no aislados y distribuidos en un poco menos de la mitad de los AGEB's de la metrópoli. Después, el otro $30 \%$ se distribuyen en espacios con barrios con presencia y presencia mínima, en la otra mitad de la metrópoli (ver Tabla 4 y Figura 4).

Por último, el nivel socioeconómico Muy Bajo generó pocos espacios de alta homogeneidad, la mayor parte se encuentran en espacios heterogéneos donde son minoría. El estrato representa el $8,3 \%$ de los jefes de familia. Primero, el $0,09 \%$ de los jefes de familia de este nivel económico viven en guetos y enclaves de exclusión, es decir, están concentrados, pero son muy pocos. Segundo, el $23 \%$ del estrato vive en enclaves no aislados, proporción significativamente menor a la de todos los demás estratos, de hecho, en todos los demás, los enclaves no aislados es su principal forma de ocupación, este grupo del estrato Bajo ocupa el 11,34\%. Tercero, a diferencia de los demás grupos poco más de la mitad de los jefes de familias del estrato aparece en barrios con presencia, es decir, representan entre el 8,3 y el $20 \%$ (ver Tabla 4 y Figura 4).

\section{Figura 4}

Guetos, enclaves de pobreza y barrios con presencia de estratos Bajo (D) y Muy Bajo (E)
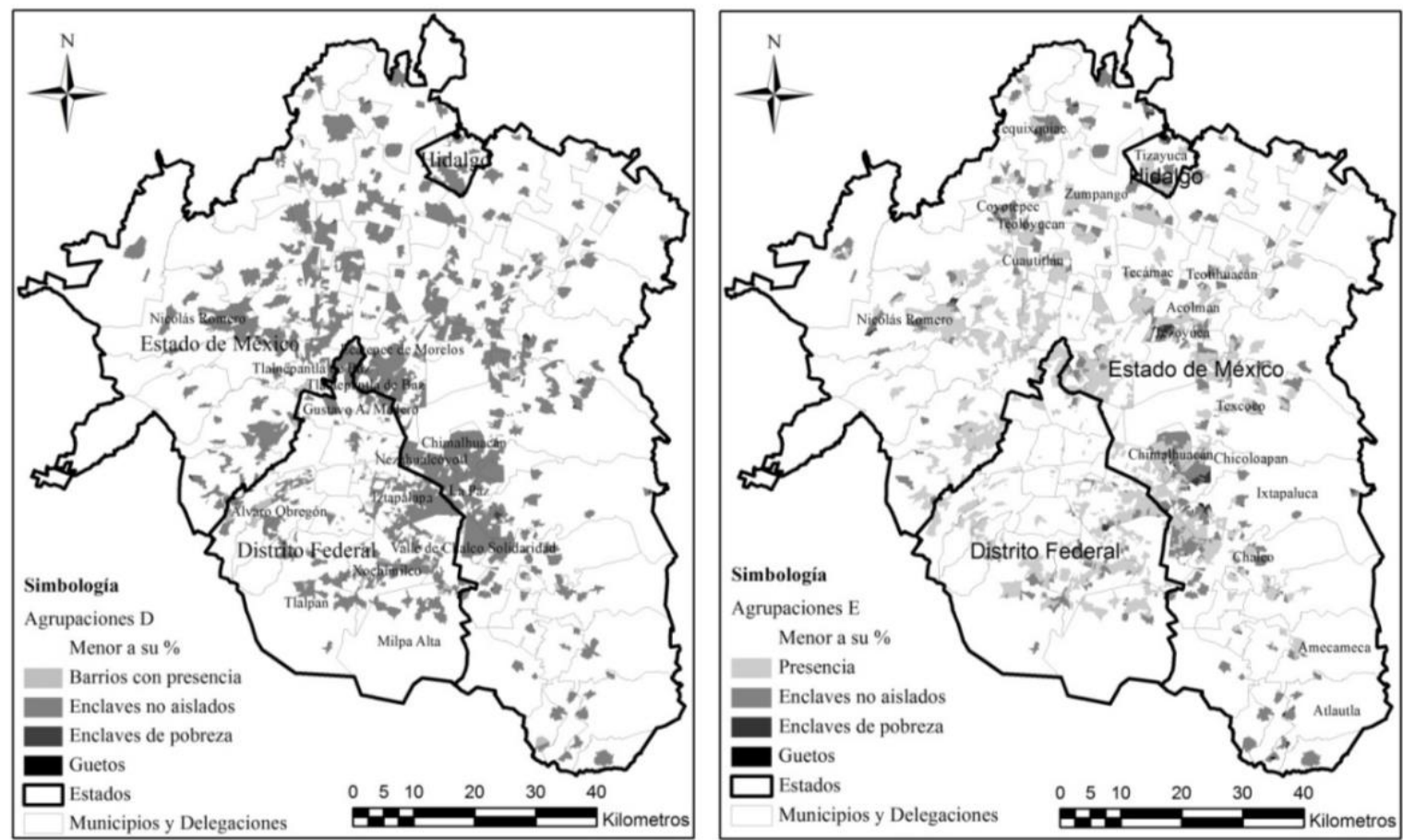

Fuente: elaboración propia, datos por AGEB construidos en el laboratorio de microdatos del INEGI utilizando el Censo de Población y Vivienda del 2010. 
Por último, el $22,9 \%$ de los hogares del estrato Muy Bajo están presentes en barrios por debajo de su porcentaje o presencia mínima en la ZMVM $(8,3 \%)$, esto es, en heterogeneidad y muy dispersos, ya que alcanzan casi el $60 \%$ de los AGEB's. Es decir, los grupos más pobres representan minorías en sus barrios y se encuentran dispersos por casi toda la ciudad.

\section{Discusión y Conclusiones}

Retomando lo planteado al inicio, de que un estudio de la segregación residencial por medio de la metodología de tipología de barrios muestra que la ZMVM no es una ciudad segregada. Nos plantea una serie de reflexiones, la primera y la más importante que se desprende de los análisis anteriores cuestiona la idea arraigada, incluso entre especialistas, de que las ciudades latinoamericanas están fuertemente segregadas como consecuencia de las profundas diferencias sociales. Por ejemplo, se asegura que se ha formado una ciudad dual a partir de la globalización, es decir que la estructura social se veía reflejada en la estructura espacial; sin embargo los resultados coinciden con lo planteado por las autoras Rubalcaba y Schteingart (2012, p. 13), quienes critican la simpleza de la idea de la "ciudad dual", que se refiere a la polarización extrema de las ciudades entre ricos y pobres, y reconocen la complejidad de la división espacial y la gradación de situaciones (pp. 14-15).

La utilización de la metodología de tipología de barrios muestra que en la ZMVM más de la mitad de la población vive en enclaves no aislados, es decir lo hace disperso en la ciudad, como minoría que convive con otros grupos el proceso parece ser el de congregación señalado por Peter Marcuse (2001), es decir la reunión voluntaria de un grupo de población sin buscar la dominación o la exclusión. Sin embargo, es importante señalar que una parte muy pequeña del grupo de mayor estatus forma ciudadelas y podría mostrar el proceso de la fortificación señalado por Marcuse (2001), es decir una reunión voluntaria con el propósito de proteger los intereses dominantes y fortalecer esa dominación. Parece que ha existido cierta laxitud en las descripciones de la segregación de algunos autores en las últimas dos décadas, empezando por reconocidos teóricos que han permeado en el pensamiento de los estudiosos del tema, quienes parecen, a su vez, haber privilegiado el discurso sobre la contrastación empírica. Si bien, no se puede decir que la segregación es un mito, si se puede asegurar que es mucho menor de lo que se percibe.

Otro hallazgo valioso de la tipología de barrios es que a diferencia de los planteamientos tradicionales de la ecología humana que afirmaban que los extremos del continuo socioeconómico son los grupos más segregados en el espacio, y olvidan a los grupos intermedios. El hecho es que para la ZMVM este planteamiento es parcialmente válido para los grupos de mayores ingresos (estrato muy alto) que, sin poderlo considerar un grupo segregado, una parte de éste sí mostró una tendencia a la fortificación formando ciudadelas y congregándose en enclaves de exclusión. Pero no resultó cierto para los grupos más pobres, que aparecieron como el estrato más disperso de todos.

Mucha de la discusión que se ha ofrecido en este apartado tiene su origen en una cuestión metodológica, como se mencionó antes, la mayor parte de los estudios sobre segregación se basan en la clasificación, generalmente multivariada de unidades espaciales a una escala de una colonia o barrio, sin embargo, el estudio de la tipología de barrios muestra que no es tal la homogeneidad de estas unidades de análisis espacial. En realidad, estos estudios ocultaban la enorme heterogeneidad social al interior de los barrios, lo que necesariamente arrojaba un patrón territorial de segregación válido de la ZMVM; pero al mismo tiempo ocultó, durante décadas, la heterogeneidad de la ciudad.

Los resultados dejan varias vetas de investigación, primero hay una cierta asociación entre los estratos socioeconómicos y los grados de homogeneidad; pero eso no se cumple en todos los niveles socioeconómicos. Segundo, será necesario profundizar en la relación entre la heterogeneidad y los ciclos de los barrios, en otras palabras, los procesos de filtrado, sucesión y gentrificación en la heterogeneidad del espacio urbano. Tercero, explicar estos procesos por medio de las formas de producción del espacio por parte de cada uno de los grupos. Cuarto, también será necesario calibrar los rangos de Johnston al caso mexicano, no necesariamente las proporciones de mixtura que él propone son las más convenientes para las ciudades latinoamericanas, y en particular, para México, en donde la mayor parte de las familias vive en enclaves no aislados. En fin, existen aún 
muchas preguntas que resolver en el tema de la segregación espacial; pero un resultado se confirma, la segregación de las ciudades, en este caso del ZMVM, es menor de lo que todos pensábamos [B]

\section{Referencias bibliográficas}

Aguilar, A. y Mateos, P. (2011). Diferenciación sociodemográfica del espacio urbano de la Ciudad de México. EURE, Revista de Estudios Urbano Regionales, 37(100), 5-30. http://dx.doi.org/10.4067/S025071612011000100001

Apparicio, P. (2000). Les indices de ségrégation résidentielle: un outil intégré dans un système d'information géographique. European Journal of Geograph, Cybergeo, 134. http://dx.doi.org/10.4000/cybergeo.12063

Ariza, M. y Solís, P. (2009). Dinámica socioeconómica y segregación espacial en tres áreas metropolitanas de México, 1990 y 2000. Estudios Sociológicos, 27(79), 171-209.

https://estudiossociologicos.colmex.mx/index.php/es /article/view/266

Arriagada, C. (2008). Segregación residencial en el tiempo de las ciudades globales: estudios comparados de las áreas metropolitanas de Chile y Canadá. En Sabatini, F., Salcedo, R., Wormald, G., y Cáceres, G. (Coords.), Tendencias de la segregación en las principales ciudades chilenas (pp. 67-92). Chile: Instituto Nacional de Estadísticas y Universidad Católica de Chile.

Azócar, G., Henríquez, C., Valenzuela, C. y Romero, H. (2008). Tendencias sociodemográficas y segregación socioespacial en Los Ángeles, Chile. Revista de Geografía Norte Grande, (41), 103-120. https://scielo.conicyt.cl/pdf/rgeong/n41/art06.pdf

Dureau, Françoise y Salas, Andrea. (2010), Las diferentes expresiones del proceso de segregación en Bogotá, en Jaramillo Samuel (ed.), Bogotá en el cambio de siglo: promesas y realidades. Quito: OLACCHI.

Garín, A., Salvo, S. y Bravo, G. (2009). Segregación residencial y políticas de vivienda en Temuco, 19922002. Revista Geografía Norte Grande, (44), 113-128. https://scielo.conicyt.cl/pdf/rgeong/n44/art06.pdf
Gómez, R. y Alvarado, C. (2016). La dinámica espacial de la segregación residencial: concentración y homogeneidad en la Zona Metropolitana del Valle de México. Cardinalis, Revista del Departamento de Geografía, 4(7), 179-197.

https://revistas.unc.edu.ar/index.php/cardi/article/vi ew/16255

Groisman, Fernando y Suárez, Ana Lourdes. (2006), Segregación residencial en la ciudad de Buenos Aires, en Población de Buenos Aires, vol. 3, núm. 4, octubre 2006, Buenos Aires: Dirección General de Estadísticas y Censos, pp. 27-37. https://www.redalyc.org/pdf/740/74030403.pdf

Johnston, R., Poulsen, M., y Forrest, J. (2003). And did the walls come tumbling down? Ethnic residential segregation in four US metropolitan areas 1980-2000. Urban Geography, 24(7), 560-581. https://doi.org/10.2747/0272-3638.24.7.560

Kaztman, R., Corbo, G., Filgueira, F., Furtado, M., Gelber, D., Retamoso, A. y Rodriguez, F. (2003). La ciudad fragmentada: mercado, territorio y marginalidad en Montevideo. Working Paper Series, Uruguay, Montevideo, Center for the Study of Urbanization and Internal Migration in Developing Countries. Population Research Center. The University of Texas at Austin. No. 2.

Linares, S. (2013). Medidas de segregación socioespacial: discusión metodológica y aplicación empírica sobre ciudades medias argentinas. Persona y Sociedad, 27 (2), 11-40.

Link, F., Valenzuela, F. y Fuentes, L. (2015). Segregación, estructura y composición social del territorio metropolitano en Santiago de Chile. Complejidades metodológicas en el análisis de la diferenciación social en el espacio. Revista de Geografía Norte Grande, (62), 151-168. https://scielo.conicyt.cl/pdf/rgeong/n62/art09.pdf 
López, H. (2009). Los niveles socioeconómicos y la distribución del gasto (Presentación). Instituto de Investigaciones Sociales S.C. AMAI.

Marcuse, P. (2001). Enclaves yes, Ghettos, no: segregation and the State. Cambridge, Massachusetts: Lincoln Institute of Land Policy.

Molinatti, F. (2013). Segregación residencial e inserción laboral en la ciudad de Córdoba. Revista de Estudios Urbano Regionales, 39(117), 117-145. https://scielo.conicyt.cl/pdf/eure/v39n117/art06.pdf

Pérez-Campuzano, E. y Santos-Cerquera, C. (2011). Diferenciación socioespacial en la Zona Metropolitana de la Ciudad de México. Investigaciones Geográficas, 72(2), 92-106.

http://www.scielo.org.mx/pdf/igeo/n74/n74a8.pdf

Peters, P. y Skop, E. (2007). Socio-spatial segregation in Metropolitan Lima, Peru. Journal of Latin American Geography, 6(1), 149-171.

Prévôt, Marie-France. (2000), Segregación, fragmentación, secesión. Hacia una nueva geografía social en la aglomeración de Buenos Aires. Economía, Sociedad y Territorio, 2(7), 405-431.

Roberts, B. (2010). Prefacio. En Sabatini, Salcedo, Wormald y Cáceres, Tendencias de la segregación en las principales ciudades Chilenas (pp. 5-7). Chile: Pontificia Universidad Católica de Chile e Instituto Nacional de Estadística.

Roberts, B. \& Wilson, R. (2009). Urban segregation and gobernance in the Americas. United States: Palgrave Macmillan.

Rodríguez, J. (2008). Movilidad cotidiana, desigualdad social y segregación residencial en cuatro metrópolis de América Latina. Revista de Estudios Urbano Regionales, 34(103), 49-71. https://scielo.conicyt.cl/pdf/eure/v34n103/art03.pdf

Rubalcaba, R. M. y Schteingart, M. (2012). Ciudades divididas: desigualdad y segregación social en México. México: El Colegio de México, Centro de Estudios Demográficos, Urbanos y Ambientales.

Sabatini, F., Cáceres, G. y Cerda, J. (2001). Segregación residencial en las principales ciudades chilenas: Tendencias de las tres últimas décadas y posibles cursos de acción. EURE (Santiago), 27(82). https://doi.org/10.4067/s0250-71612001008200002
Sabatini, F. y Sierralta, C. (2006). Medição da segregação residencial: meandros teóricos e metodológicos e especificidade latino-americana. En Pinto da Cunha, José Marcos (Editor), Novas Metrópoles Paulistas; População, Vulnerabilidade e Segregação (pp. 169195). Brasil: Nepo-Unicamp, Campinas.

Sabatini, F., Cáceres, G., Sierralta, C. y Robles, S. (2008). La segregación residencial en cinco ciudades chilenas según las estadísticas censales: tendencias y giros. En Sabatini, F., Salcedo, R., Wormald, G., y Cáceres, G. (coords.), Tendencias de la segregación en las principales ciudades chilenas; análisis censal 19822002 (pp. 9-18). Chile: Instituto Nacional de Estadísticas y Universidad Católica de Chile.

Sabatini, F., Wormald, G., Sierralta, C. y Peters, P. (2008). Segregación Residencial en Santiago: Tendencias 1992-2002 y efectos vinculados con su escala geográfica. En Sabatini, F., Salcedo, R., Wormald, G., y Cáceres, G. (coords.), Tendencias de la segregación en las principales ciudades chilenas; análisis censal 19822002 (pp. 19-42). Chile: Instituto Nacional de Estadísticas y Universidad Católica de Chile.

Sánchez, L. (2012). Cambios en la segregación residencial socioeconómica en México. Realidad, datos y espacio. Revista internacional de estadística y geografía, 3(2), 98-115.

https://www.inegi.org.mx/rde/RDE_06/Doctos/RDE 06 opt.pdf

Saravi, G. (2004). Segregación urbana y espacio público: los jóvenes en enclaves de pobreza estructural. Revista de la CEPAL, (83), 33-48. https://repositorio.cepal.org/handle/11362/10962

Thais, R., Bichir, R y Moller-Holtkamp, T. (2008). Patrones de segregación en perspectiva comparada: los casos de Sao Paulo y Santiago de Chile. En Sabatini, F., Salcedo, R., Wormald, G., y Cáceres, G. (coords.), Tendencias de la segregación en las principales ciudades chilenas; análisis censal 1982-2002 (pp. 4366). Chile: Instituto Nacional de Estadísticas y Universidad Católica de Chile.

Wacquant, Loïc. Las dos caras de un gueto. La construcción de un concepto sociológico. Renglones, (56), 72-80. http://renglones.iteso.mx/upload/archivos/56 11 do s caras.pdf 